\title{
EVERY NUMERICAL SEMIGROUP IS ONE HALF OF A SYMMETRIC NUMERICAL SEMIGROUP
}

\author{
J. C. ROSALES AND P. A. GARCÍA-SÁNCHEZ
}

(Communicated by Martin Lorenz)

\begin{abstract}
Let $S$ be a numerical semigroup. Then there exists a symmetric numerical semigroup $\bar{S}$ such that $S=\{n \in \mathbb{N} \mid 2 n \in \bar{S}\}$.
\end{abstract}

Let $\mathbb{N}$ be the set of nonnegative integers. A submonoid $M$ of $\mathbb{N}$ is a subset of $\mathbb{N}$ closed under addition and such that $0 \in M$. A numerical semigroup $S$ is a submonoid of $\mathbb{N}$ such that $\mathbb{N} \backslash S$ is finite. The elements of $\mathrm{H}(S)=\mathbb{N} \backslash S$ are the gaps of $S$, and the largest integer not belonging to $S$ is known as the Frobenius number of $S$, denoted here by $\mathrm{F}(S)$. There is a huge amount of papers devoted to the Frobenius number and more particulary to the problem of computing or bounding it (see [1] and the vast list of references given there).

A numerical semigroup $S$ is said to be symmetric if for every $z \in \mathbb{Z}$, either $z \in S$ or $\mathrm{F}(S)-z \in S$. Symmetric numerical semigroups earned some relevance due to a result by Kunz (see [2]), which states that the semigroup ring of a symmetric numerical semigroup is Gorenstein. Thus this class of numerical semigroups became a source of examples of Gorenstein rings.

For a numerical semigroup $S$, define $\mathrm{N}(S)=\{s \in S \mid s<\mathrm{F}(S)\}$. Clearly, $\# \mathrm{~N}(S)+\# \mathrm{H}(S)=\mathrm{F}(S)+1$, and $S$ is symmetric if and only if $\# \mathrm{~N}(S)=\# \mathrm{H}(S)$, where \#A denotes the cardinality of $A$.

Let $M$ be a submonoid of $\mathbb{N}$ and let $d$ be a positive integer. Then

$$
\frac{M}{d}=\{n \in \mathbb{N} \mid d n \in M\}
$$

is again a submonoid of $\mathbb{N}$, called the quotient of $M$ by $d$. Clearly, $M \subseteq \frac{M}{d}$.

The aim of this paper is to show that for every numerical semigroup $S$, there exists a symmetric numerical semigroup $\bar{S}$ such that $S=\frac{\bar{S}}{2}$.

Given $A$, a subset of $\mathbb{Z}$, denote by $2 A=\{2 a \mid a \in A\}$ (not to be confused with $A+A)$. Given $x_{1}, \ldots, x_{k} \in \mathbb{Z}$, when we write $\left\{x_{1}, \ldots, x_{k}, \rightarrow\right\}$, we mean that all the integers greater than $x_{k}$ belong to the set.

Received by the editors January 19, 2007.

2000 Mathematics Subject Classification. Primary 20M14, $13 \mathrm{H} 10$.

Key words and phrases. Numerical semigroup, symmetric numerical semigroup, Frobenius number.

The authors were supported by the project MTM2004-01446 and FEDER funds.

The authors want to thank the referee for her/his comments and suggestions. 
Theorem 1. Let $S$ be a numerical semigroup. Then there exists a symmetric numerical semigroup $\bar{S}$ such that

$$
S=\frac{\bar{S}}{2}
$$

Proof. Let $g=\mathrm{F}(S)$. If $g=-1$, then $S=\mathbb{N}$. Clearly $\mathbb{N}=\frac{\mathbb{N}}{2}$ and $\mathbb{N}$ is symmetric. Now assume that $g=1$. In this setting $S=\{0,2, \rightarrow\}$. The reader can easily check that by taking $\bar{S}=\{0,4,5,6,8, \rightarrow\}$, one gets that $S=\frac{\bar{S}}{2}$, with $\bar{S}$ symmetric.

Now suppose that $g \geq 2$. Let $\mathrm{H}(S)=\left\{h_{1}, \ldots, h_{t}\right\}$. We claim that

$$
\bar{S}=2 S \cup\left\{2 g+\left(2 g-2 h_{1}\right)-1, \ldots, 2 g+\left(2 g-2 h_{t}\right)-1\right\} \cup\{4 g, \rightarrow\}
$$

is a symmetric numerical semigroup and that $S=\frac{\bar{S}}{2}$.

First we must prove that $\bar{S}$ is a submonoid of $\mathbb{N}$. This is a consequence of the following remarks.

1. $2 S$ is a submonoid of $\mathbb{N}$.

2. The result of adding any nonnegative integer to any element in $\{4 g, \rightarrow\}$ remains in $\{4 g, \rightarrow\}$.

3. For every $i, j \in\{1, \ldots, t\}$, we have that $2 g+\left(2 g-2 h_{i}\right)-1+2 g+\left(2 g-2 h_{j}\right)-1=$ $2\left(2 g+\left(g-h_{i}\right)+\left(g-h_{j}\right)-1\right)$. As $g=\max \left\{h_{1}, \ldots, h_{t}\right\}, 2 g+\left(g-h_{i}\right)+\left(g-h_{j}\right)-1 \geq$ $2 g-1$. Since by hypothesis $g \geq 2$, we deduce that $2 g+\left(g-h_{i}\right)+\left(g-h_{j}\right)-1 \geq g+1$. Hence $2 g+\left(g-h_{i}\right)+\left(g-h_{j}\right)-1 \in S$, and $2 g+\left(2 g-2 h_{i}\right)-1+2 g+\left(2 g-2 h_{j}\right)-1 \in$ $2 S \subseteq \bar{S}$.

4. Now take $s \in S \backslash\{0\}$ and $i \in\{1, \ldots, t\}$. We distinguish two cases.

- If $s>h_{i}$, then $2 g+\left(2 g-2 h_{i}\right)-1+2 s=4 g+2\left(s-h_{i}\right)-1 \geq 4 g$, whence $2 g+\left(2 g-2 h_{i}\right)-1+2 s$ belongs to $\bar{S}$.

- If $s<h_{i}$, then $0 \leq g+\left(s-h_{i}\right)<g$. Thus $g+s-h_{i}=g-x$, with $x=h_{i}-s \in\{1, \ldots, g\}$. As $h_{i}=s+x \notin S$, and $s \in S$, we have that $x \notin S$. Hence $x=h_{j}$ for some $j \in\{1, \ldots, t\}$. This leads to $2 g+\left(2 g-2 h_{i}\right)-1+2 s=$ $2 g+\left(2 g-2 h_{j}\right)-1 \in \bar{S}$.

Since $\{4 g, \rightarrow\} \subseteq \bar{S}$, we have that $\mathbb{N} \backslash \bar{S}$ has finitely many elements. This proves that $\bar{S}$ is a numerical semigroup.

Observe that $4 g-1$ is odd. As $\bar{S}=2 S \cup\left\{2 g+\left(2 g-2 h_{1}\right)-1, \ldots, 2 g+(2 g-\right.$ $\left.\left.2 h_{t}\right)-1\right\} \cup\{4 g, \rightarrow\}$ and the elements in $2 S$ are all even, if $4 g-1 \in \bar{S}$, then $4 g-1=2 g+\left(2 g-2 h_{i}\right)-1$ for some $i \in\{1, \ldots, t\}$. But this leads to $h_{i}=0$, which is impossible. Hence $4 g-1=\mathrm{F}(\bar{S})$, because $\{4 g, \rightarrow\} \subseteq \bar{S}$.

In order to prove that $\bar{S}$ is symmetric, let us show that $\# \mathrm{~N}(\bar{S})=\# \mathrm{H}(\bar{S})$. By using that $\mathrm{F}(\bar{S})=4 g-1$, we easily deduce that

$$
\begin{aligned}
\mathrm{N}(\bar{S})=2 \mathrm{~N}(S) \cup\{2(g+1), 2(g+2), \ldots, 2(g+(g-1))\} & \\
& \cup\left\{2 g+\left(2 g-2 h_{1}\right)-1, \ldots, 2 g+\left(2 g-2 h_{t}\right)-1\right\}
\end{aligned}
$$

(and that these sets are disjoint). Hence $\# \mathrm{~N}(\bar{S})=\# \mathrm{~N}(S)+g-1+\# \mathrm{H}(S)$. As we know that $g+1=\# \mathrm{H}(S)+\# \mathrm{~N}(S)$, we obtain that $\# \mathrm{~N}(\bar{S})=2 g$. We also know that $4 g-1+1=\# \mathrm{H}(\bar{S})+\# \mathrm{~N}(\bar{S})$, and consequently $\# \mathrm{H}(\bar{S})=2 g=\# \mathrm{~N}(\bar{S})$.

Finally, we show that $S=\frac{\bar{S}}{2}$. As $2 S \subseteq \bar{S}$, the inclusion $S \subseteq \frac{\bar{S}}{2}$ is trivial. For the other inclusion, let $x \in \frac{\bar{S}}{2}$. Then $2 x \in \bar{S}$. From the way $\bar{S}$ is defined, as $2 x$ is even, either $2 x \in 2 S$ or $2 x \geq 4 g$. If $2 x \in 2 S$, then trivially $x \in S$. If $2 x \geq 4 g$, then $2 x>2 g$ and thus $x>g$, which also leads to $x \in S$. 


\section{References}

[1] J. L. Ramírez Alfonsín, The Diophantine Frobenius problem, Oxford Lecture Series in Mathematics and Its Applications, 30. Oxford University Press, Oxford, 2005. MR02260521 (2007i:11052)

[2] E. Kunz, The value semigroup of one dimensional Gorenstein ring, Proc. Amer. Math. Soc. 25 (1970), 748-751. MR0265353 (42:263)

Departamento de Álgebra, Universidad de Granada, E-18071 Granada, Spain

E-mail address: jrosales@ugr.es

Departamento de Álgebra, Universidad de Granada, E-18071 Granada, Spain

E-mail address: pedro@ugr.es 